\title{
Age-Related Retinal Vasculopathy
}

\author{
W. R. LEE, G. E. BLASS and D. C. SHAW \\ Glasgow
}

\begin{abstract}
Summary
Second and third order retinal arterioles and venules were studied by immunohistochemistry and electron microscopy in the uncomplicated ageing process and in association with central retinal vascular occlusive disease (CRVOD). Tissue was obtained from eight enucleated human eyes (4 control, 4 with manifestations of CRVOD) and the investigation was directed towards abnormalities in the myocytes and the nature of collagenous materials which are deposited during the process of hyalinsation. In normal ageing and in two cases of CRVOD, the endothelial cell monolayer and the underlying cells (subendothelial myocytes) were preserved in arterioles and venules: there was no evidence of fibrin leakage. Degenerative changes were found in the medial myocytes in control tissue from the fifth decade and these included myocyte shrinkage, accumulation of intracytoplasmic membranous structures, cytoplasmic vacuolation and fragmentation. The 'hyalinised' acellular vessel wall seen in CRVOD contains scattered activated fibroblast-like myocytes and macrophages lying within a matrix of fibronectin, $65 \mathrm{~nm}$ collagen and multilayered basement membrane material. Endothelial cells and cohort subendothelial myocytes are involved in the formation of capillaries which bud into the hyalinised vessel wall in CRVOD. In two cases of CRVOD there was extensive cellular degeneration and cell debris accumulated within the degenerate stroma: this was attributed to superadded total ischaemia. The pathogenesis of hyalinisation remains obscure but a subtle age-related dysfunction of the morphologically intact lining endothelium could be responsible for metabolic damage to myocytes in the media.
\end{abstract}

Secondary degenerative disease in the retinal vasculature (due to e.g. hypertension and central retinal vascular occlusive disease (CRVOD)) has been studied extensively by light microscopy, ${ }^{1-5}$ and to a lesser extent by electron microscopy. ${ }^{6}$ The neglect of the simple process of age-related vasculopathy makes it difficult to form valid assessment of secondary disease as studied by the techniques of electron microscopy and immunohistochemistry. Recent light microscopic studies, ${ }^{4,5}$ have highlighted the importance of vasculosclerosis (hyalinisation) in retinal neo- vascularisation and have stressed the frequent association of this abnormality with CRVOD. In the course of further morphological background research into the problems of neovascularisation associated with degenerative vasculopathy, we encountered significant changes in control tissue in addition to unexpected findings in diseased vessels. These abnormalities have not been reported to our knowledge and are the subject of this presentation.

\section{Material and Methods}

Trapezoidal blocks of tissue which included second

Address for correspondence: Professor W. R. Lee, Pathology Department, Western Infirmary, Glasgow G11 6NT.

From: The Departments of Ophthalmology and Pathology, University of Glasgow. 
Table I Relevant pathology in enucleation specimens

\begin{tabular}{|c|c|c|c|}
\hline Case No. & \multicolumn{2}{|c|}{ Age/Sex } & Relevant pathological findings \\
\hline \multicolumn{4}{|l|}{$\begin{array}{l}\text { 'Control' } \\
\text { tissue }\end{array}$} \\
\hline 1 & $2 \frac{1}{2}$ & $\mathrm{~F}$ & inferior endophytic retinoblastoma superior retina not involved \\
\hline 2 & 52 & M & $\begin{array}{l}\text { superotemporal melanoma } \\
(15 \times 10 \times 10 \mathrm{~mm}) \\
\text { inferior retina detached by exudate }\end{array}$ \\
\hline 3 & 60 & M & inferior choroidal melanoma, retina in situ above \\
\hline 4 & 67 & $\mathbf{F}$ & exenteration for carcinoma of ethmoid \\
\hline \multicolumn{4}{|c|}{ Advanced vasculopathy in CRVOD and neovascular glaucoma } \\
\hline 5 & 83 & $\mathrm{~F}$ & intramural capillary ingrowth \\
\hline 6 & 55 & $\mathbf{F}$ & intramural ingrowth and intraretinal neovascularisation \\
\hline 7 & 85 & $\mathrm{~F}$ & intraretinal nodules in blood vessels: total acellularity \\
\hline 8 & 70 & $\mathbf{F}$ & pre-retinal neovascular nodules total acellularity in hyalinised tissue \\
\hline
\end{tabular}

and third order arterioles and venules, were removed from the retina in eight surgically enucleated eyes. (for details see Table I). Primary fixation was by immersion in 2 per cent glutaraldehyde in cacodylate buffer. The retina was examined at high magnification with an Olympus Stereomicroscope and photomacrography was performed where appropriate. In control tissue, the ostensibly normal vessels and surrounding retina were removed at random. In the globes removed in treatment of neovascular glaucoma and CRVOD, regions in which there was detection of intramural intraretinal or pre-retinal neovascularisation were also studied. The blocks were taken so that the venules and arterioles were sectioned transversely.

After routine processing for paraffin embedding, serial sections were used for three dimensional reconstruction, sections were also stained to demonstrate the presence or absence of fibrin and collagen (MSB and trichromes), reticulin (Gordon and Sweet's) and elastic material (Elastic van Gieson). For carbohydrate histochemical analysis, PAS \pm diastase, Alcian blue-PAS and Alcian blue \pm hyaluronidase were utilised. Immunohistochemical techniques were employed to detect the presence of Factor VIII, fibronectin and fibrinogen, by means of antibodies raised in rabbits (Dako). Enzyme pretreatment was required (0.1 per cent Trypsin) prior to labelling with the unlabelled antibody peroxidase-antiperoxidase technique.

Three-dimensional reconstructions were made by overlaying tracing paper onto serial photomicrographs of vessels in which there was intramural capillary formation. An $0.5 \mathrm{~cm}$ overlap was made between each tracing, using one fixed feature as a reference point.

Tissue blocks were processed for araldite embed- ding after osmication. In some cases semi-thin sections (toluidine blue stained) were cut serially, with intervening ultrathin section preparation. The ultrathin sections were stained with uranyl acetate and lead citrate for examination in a Philips 301 electron microscope.

\section{Results}

\section{Light microscopy}

In control tissue in the infantile and adult retina, arterioles and venules are easily distinguished. The former has a thicker wall with four to eight layers of smooth muscle cells surrounding an attenuated endothelial layer. The muscle layers of the venular wall are only two in number. The endothelium showed a positive reaction with Factor VII antibody, but reactions to fibronectin and fibrinogen were negative.

In the hyalinised vessels studied by histochemistry in three eyes which showed the pathology of CRVOD, ${ }^{4,5}$ the stroma demonstrated a positive immunohistochemical reaction for fibronectin and a negative reäction for fibrinogen.

Serial sections of hyalinised vessels which contained intramural capillaries (Fig. 1) revealed that the channels communicated with the parent lumen which remained at a constant internal diameter throughout. Serial reconstructions revealed bifurcations, $T$ channels and blind channels. Special tinctorial stains to demonstrate the presence of fibrin or fibrinogen (e.g. MSB) were negative in these regions of intramural neovascularisation and 

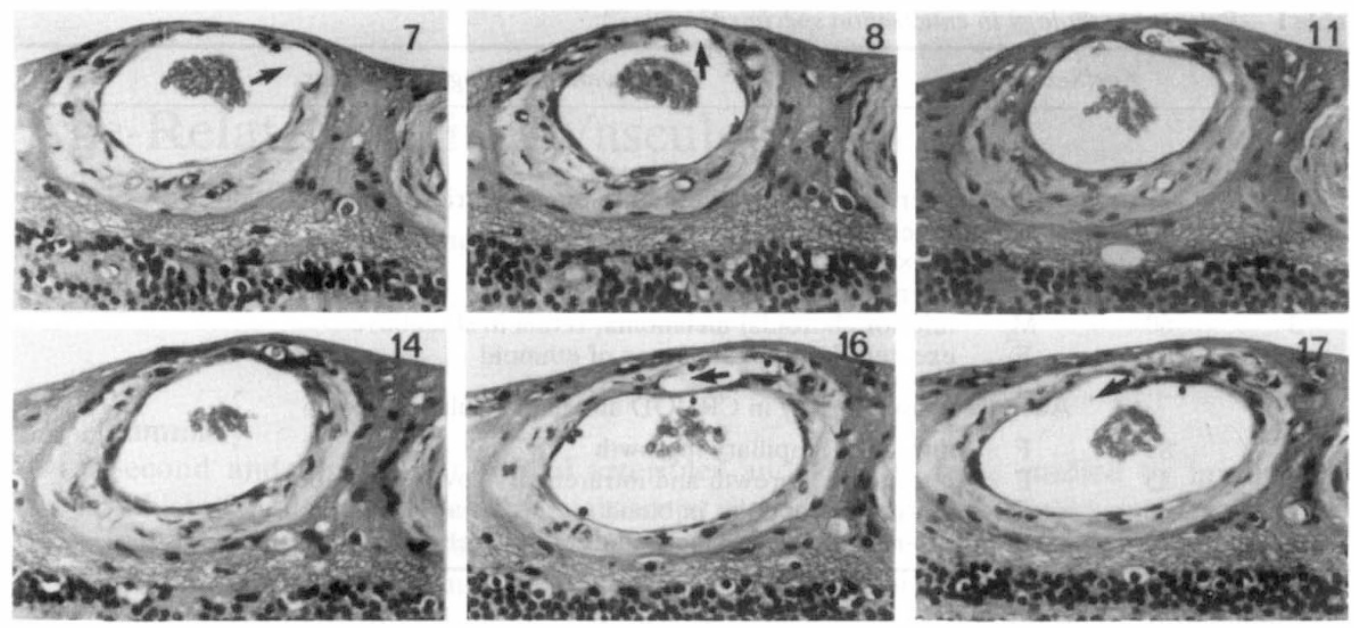

Fig. 1. Serial sections (levels indicated top right) along a hyalinised vessel to show the course of an intramural capillary ( $\uparrow)(H \& E \times 120)$.

only those designed to demonstrate the presence of basement membrane and collagen were positive in hyalinised blood vessels.

The electron microscopic investigation of uncomplicated ageing in blood vessels revealed interesting changes in two casesboth male, aged 52 and 60 years respectively. The endothelium was unremarkable and on no occasion was there evidence to indicate any abnormality in the intercellular junctions. Subendothelial myocyte architecture was well-preserved. In some arterioles the medial myocytes showed cytoplasmic rarefaction with organelle degeneration and intracytoplasmic membranous debris (Fig. 2a). In a 52 year old patient, the arterioles sampled showed an apparent patchy absence of myocytes associated with extensive multilayering of basement membrane and deposition of $65 \mathrm{~nm}$ collagen (Fig. 2b). The remaining myocytes were enlarged and the cytoplasm contained plentiful rough endoplasmic reticulum in addition to persisting submembranous densifications of actin filaments (Fig. 2c). A noteworthy feature was that fragments of cell debris were present between the layers of basement membrane. Macrophages containing small secondary lysosomal bodies were found within the walls of vessels in which there was an apparent depletion of myocytes (Fig. 2d). Sometimes Muller cell processes protruded into the walls of the hyalinised vessels.

\section{Ultrastructural changes in retinal vessels in CRVOD}

The consistent basic feature of the vasculopathy studied in four cases (Table I) was that the thickened vessel walls contained very few, if any, medial myocytes (Fig. 3a). The wall consisted of multilayered basement membrane and collagen fibres within which macrophages were identified. This abnormality was such that it was not possible to differentiate between degenerate arterioles and venules, a feature which has been emphasised in previous light microscopic studies. ${ }^{4.5}$ In two cases, however, the endothelial cells and the underlying subendothelial myocytes were preserved. One case provided the opportunity to study intramural neovascularisation and examples were found which showed an invagination from the lumen (Fig. 3b) and the presence of capillaries within the accellular connective tissue: this corresponded to the appearance of the tissue as demonstrated in Figure 1. The most important feature of this phenomenon was that the endothelial cell was invariably accompanied by a cohort spindle cell which had a less dense cell cytoplasm, but little else in the way of identifying features (Fig. 3c). Nevertheless, continuity with surviving subendothelial myocytes suggested 

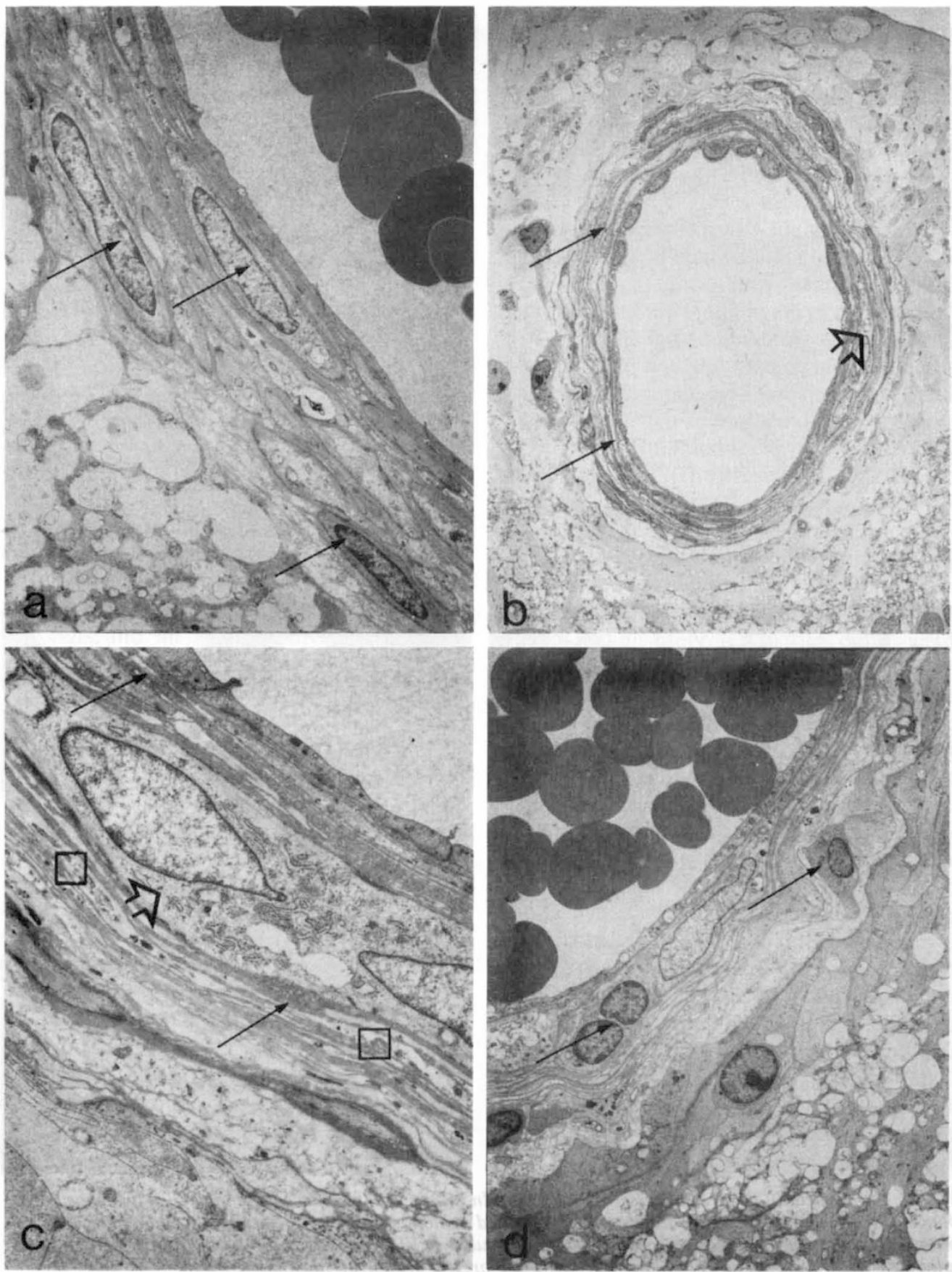

Fig. 2. Ultrastructural features of arterioles in the fifth and sixth decades. In (a) some myocytes show normal architecture ( $\uparrow)$, but there is evidence of cytoplasmic degeneration (open arrows) in other cells (60M). In (b) and (c) the myocytes are replaced by multilayered basement membrane ( $\uparrow$ ) and collagen, with some persisting transformed myocytes (open arrows). Note cell debris ( $\square)$. In d) macrophages ( $\uparrow$ ) are present between the layers of basement membrane and collagen.

(a) $\times 2470 ; b) \times 770 ;(c) \times 5400 ;(d) \times 2340)$ 

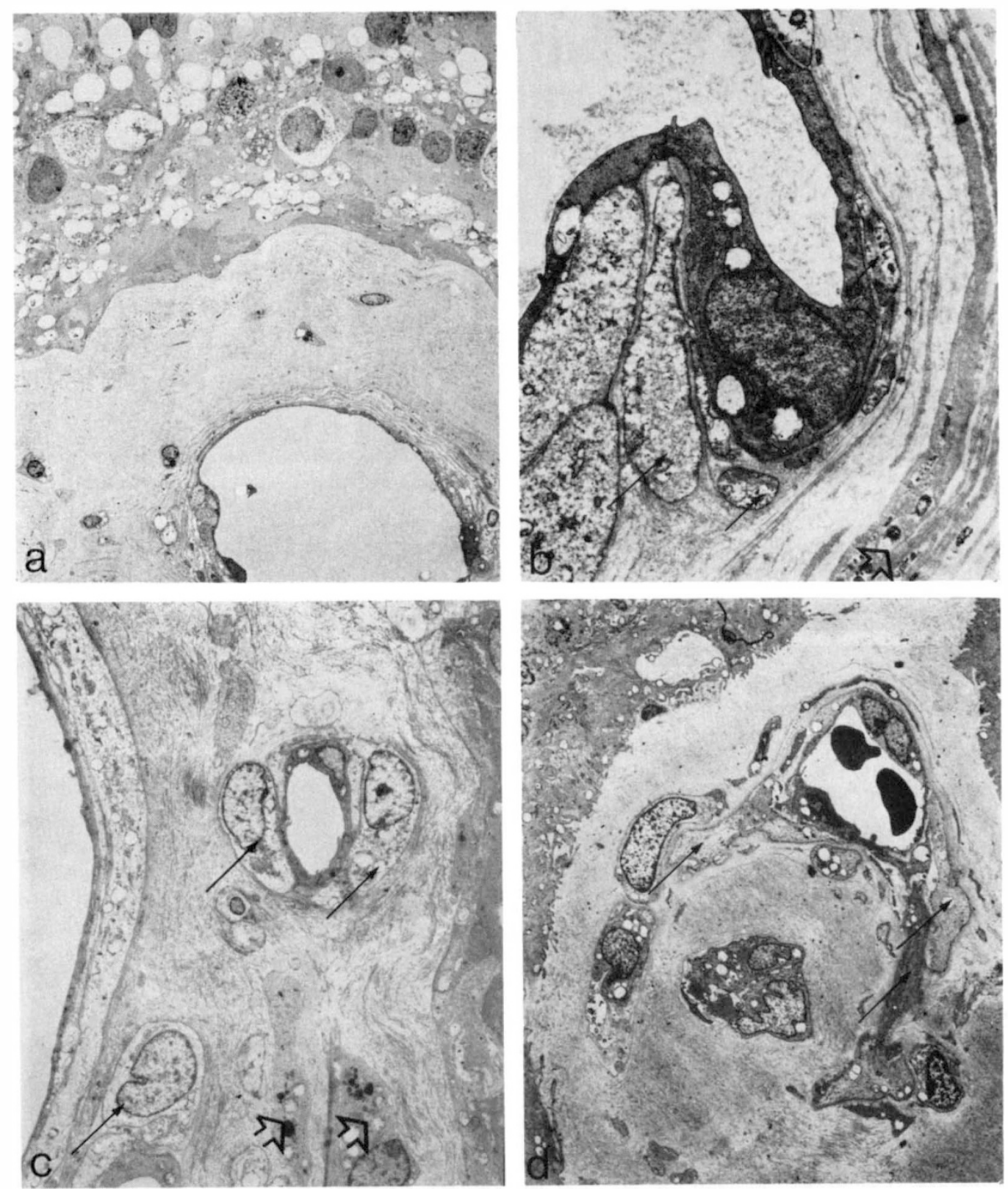

Fig. 3. Ultrastructural features of vessels in CRVOD. In (a) the wall is replaced and thickened by deposition of collagen and basement membrane and it contains scattered mononuclear macrophages. In $(b)$ endothelial cells with cohort subendothelial myocytes $(\uparrow$ ) project into the acellular vessel wall containing cell debris (open arrow). In (c) endothelial cells surrounded by cohort cells $(\uparrow)$ are present within the hyalinised wall, which also contains macrophages (open arrows).

In (d) endothelial cells and cells with characteristics of smooth muscle cells $(\uparrow)$ ramify in hyalinised connective tissue.

(a) $\times 600 ;(b) \times 7200 ;(c) \times 4500 ;(d) \times 1500)$. 
that at the site of invagination at least, transformed myocytes contributed to the cohort cell population.

In one specimen, sections through a focus of neovascularisation within a hyalinised occluded vessel were obtained. These consisted of a cluster of endothelial cells, which were joined by intact intercellular junctions and were surrounded by cohort spindle cells (Fig. 2d). There was no evidence of a continuous basement membrane around the cohort cells, so that the arrangement was not that of the normal pericyte-endothelial cell relationship. An important feature of renewed cellular activity within the collagenised vessel wall, was the presence of cells which had the characteristics of macrophages. These cells had long processes and were not surrounded by a basement membrane: the cytoplasm contained secondary lysosomes.

In the retina in two globes $(70 \mathrm{~F}$ and $85 \mathrm{~F}$ ) there was extensive degeneration of endothelial cells and an absence of subendothelial myocytes. The laminated bundles of collagen and basement membrane contained granular fragments of cell debris. This end-stage pathology was found within intraretinal nodules and in pre-retinal new vessels: the light microscopic characteristics of this have been discussed previously. ${ }^{4,5}$ In these specimens, there was extensive degeneration in the adjacent retinal neuronal tissue with the formation of intercellular spaces. It seemed reasonable to assume that the stem vessel supplying these acellular hyalinised vessels had been totally obstructed.

\section{Discussion}

The demonstration of myocyte death and fibrous replacement (hyalinisation) in the walls of retinal vessels during the ageing process in this study and that of other workers ${ }^{2,6}$ may on superficial consideration, appear to represent an innocuous process. It could be assumed that the functional effect would be simply a luminal dilatation and loss of vascular tonus. Indeed this is probably the situation in the majority of individuals and it has been suggested $^{2}$ that the loss of contractile myocytes, would have a protective effect should malignant hypertension supervene in the elderly. However, in one previous study ${ }^{6}$ and in the present study, vasculosclerosis appears to cause luminal narrowing and when Poiseuille's law is taken into consideration, the effect on tissue perfusion may be more significant than is generally appreciated ${ }^{4}$ Certainly, vasculosclerosis is an almost inevitable association with CRVOD in the enucleated eye, which adds weight to the suggestion of Wise ${ }^{3}$ and Rubenstein ${ }^{7}$ that vascular underperfusion and reduced blood flow are precipitating factors in central retinal vein occlusion.

One of the most interesting features of the investigation was the ultrastructural demonstration of degeneration and drop out of individual myocytes in the media of arterioles which by light microscopy appeared to be 'unremarkable'. The presence of multilayered basement membrane suggests two possibilities. The first is that this was the residue of an extracellular skeleton which previously surrounded myocytes: this morphological study suggests that the cells which had degenerated had disintegrated to form debris which had been phagocytosed by macrophages. The second possibility is that the basement membrane material was secreted either by endothelial cells, or by surviving subendothelial myocytes. It is also possible that mural myocytes may have transformed to become activated myofibroblasts. The latter phenomenon has been extensively studied by Staubesand and his colleagues (for review see Staubesand $^{8}$ ) who emphasised the metabolically synthetic capabilities of transformed myocytes (m-cells). The presence of a large quantity of fibrillar collagen (Type I) which was intermingled with basement membranes implies the presence of cells with adopted fibroblastic function.

The cause of selective cell death within the population of medial myocytes is unknown, but it is tempting to suggest that this is a reflection of focal and or transient vascular underperfusion. It is relevant to mention the experimental work of Juarez et al. ${ }^{9}$ who occluded the central retinal artery and vein simultaneously in young monkeys. The retinal arterioles initially ( 9 days post occlusion) showed degenerative changes similar to those described in the present study. Later (4 weeks post occlusion) the media was necrotic and the vessel 'recanalised' by endothelial cells and 
cohort pericytes. While this extreme and acute insult was obviously not applied to the human tissue in this investigation, extrapolation may be justified. An important finding in the cited experimental study, ${ }^{9}$ was that horseradish peroxidase did not leak between the tight junctions of the endothelium and it is noteworthy that in all but two of the human eyes used for this study, the endothelial architecture and intercellular attachments were preserved. In cases 7 and 8, there was obviously an extreme superadded ischaemic episode which led to total necrosis of all the surviving cellular components of the vessel wall. Fibrin or fibrin degradation products were not identified by electron microscopy within the subendothelium or within the media in any of the specimens examined, so this form of intimal thickening in degenerative vasculopathy may differ from the extreme process of fibrin leakage through a damaged endothelium as has been described in malignant hypertension..$^{1,2,10,11}$ It is not acceptable however, to exclude the possibility that there had been leakage of plasma constituents across the endothelium at some stage in the disease process.

Possibly the most intriguing aspect of the study is the relationship between hyalinisation and neovascularisation when the ageing process is complicated by CRVOD. ${ }^{4.5}$ In the mildest form endothelial cells and subendothelial myocytes invade the hyalinised connective tissue to form intramural capillaries, an abnormality which was initially described by Lowenstein ${ }^{12}$ in 1948 . The paradox is that while mild and slowly progressive ischaemia and ageing lead to myocyte death, the superadded ischaemic insult of central vein occlusion appears to stimulate endothelial cell and myocyte proliferation.

The factors which stimulate endothelial cell proliferation in vitro are under extensive investigation by cell biologists at the present time and a detailed discussion of the possible stimuli in degenerative vasculopathy would be unacceptably speculative. In relation to the morphologic findings in the present investigation, fibronectin, fibroblast-like cells, and macrophages were found within hyanalinised vessel walls which was surrounded by retinal tissue which showed the changes characteris- tic of hypoxic damage. These features individually and in combination are known from in vitro studies and pathological studies to serve as stimuli to cell proliferation. ${ }^{13-17}$ What is perhaps the most important feature of the study was the demonstration by electron microscopy of the close relationship between endothelial cells and cohort cells, which seem most likely to be derived from transformed subendothelial myocytes. This pathomorphology suggests that the in vitro models for endothelial cell proliferation should take into account that in vivo, two or more cell populations are involved.

It is a pleasure to acknowledge the technical assistance provided by Mrs S. A. Cameron, Mrs D. A. Aitken and $\mathrm{Mr}$ J. A. W. Ralston, FIMLS. The senior author is indebted to Professor Willem A. Manschot of the Erasmus University of Rotterdam, for providing the stimulus for this research.

\section{References}

${ }^{1}$ Ashton N: The eye in malignant hypertension. Trans Am Acad Ophthalmol Otolaryngol 1972; 76: 17-40.

${ }^{2}$ Leishman $\mathrm{R}$ : The eye in general vascular disease. Hypertension and arteriosclerosis. $\mathrm{Br} \quad J$ Ophthalmol 1957; 41: 641-701.

${ }^{3}$ Wise GN: Retinal arteriosclerosis secondary to vein obstruction. Arch Ophthalmol 1959; 61: 766-79.

${ }^{4}$ Manschot WA, Lee WR: Retinal neovascularisation arising from hyalinised blood vessels. Graefe's Arch clin exp Ophthal 1984; 222: 63-70.

${ }^{5}$ Manschot WA, Lee WR: Development of retinal neovascularisation in vascular occlusive disease. Trans Ophthalmol Soc UK 1985; 104: 880-86.

${ }^{6}$ Kohno T, Ishibashi T, Inomata H: Electron microscopic studies of pipestem sheathed vessels in human retina. Jap J Ophthalmol 1983; 27: 22835.

${ }^{7}$ Rubenstein K: Arterial insufficiency in retinal venous occlusion. Trans Ophthalmol Soc UK 1964; 84: 564-81.

${ }^{8}$ Staubesand J: Mediadysplasia und Arteriosklerose. Therapie Wache 1982; 32: 951-877.

${ }_{9}^{9}$ Juarez CP, Tso MOM, van Heuven AJ, Hayreh MS, Hayreh SS: Experimental vascular occlusion. III. An ultrastructural study of simultaneous occlusion of central retinal vein and artery. Int Ophthalmol 1986; 9: 89-101.

${ }^{10}$ Lendrum AC: The hypertensive diabetic kidney as a model of the so-called collagen diseases. Canad Med Assoc J 1963; 88: 442-52.

${ }^{11}$ Gardner DL, Mathews MA: Ultrastructure of the wall of small arteries in early experimental rat hypertension. J Pathol 1969; 97: 51-62.

${ }^{12}$ Lowenstein A: Intramural vascular system (vasa 
vasorum?) in retinal vessels. Arch Ophthalmol 1948; 9-26.

${ }^{13}$ Thorgeirrson G, Roberston AL: The vascular endothelium-pathologic significance. Am J Pathol 1978; 93: 803-47.

${ }^{14}$ Henkind P: Ocular neovascularisation. Am J Ophthalmol 1978; 85: 287-301.

${ }^{15}$ Madri JA, Steen KS: Aortic endothelial cell migra- tion. 1. Matrix requirements and composition. Am J Pathol 1982; 106: 180-6.

${ }^{16}$ Delvos V, Gajdnsek C, Sage H, Harker LA, Schwartz SM: Interactions of vascular wall cells with collagen gels. Lab Invest 1982; 46: 61-72.

${ }_{17}$ Abrahamson DR: Recent studies on the structure and pathology of basement membrane. J Pathol 1985; 149: 257-78. 